\title{
The back-and-forth method: A quick and simple technique for reconstitution of injectable poly-D,L-lactic acid
}

\author{
Se-Yi Chen ${ }^{1,2}$, Jui-Yu Lin ${ }^{3}$, \\ Chuan-Yuan Lin $^{3}$ \\ ${ }^{1}$ Department of Neurosurgery, Chung- \\ Shan Medical University Hospital, \\ Taichung; ${ }^{2}$ School of Medicine, Chung- \\ Shan Medical University, Taichung, \\ ${ }^{3}$ Tobeauty Aesthetic Clinic, Taipei, Taiwan
}

The authors want to give special thanks to Ella Yi-Hsin Yen (But Design Studio, Taipei, Taiwan) for her kind assistance with video editing.
Injectable poly-D,L-lactic acid (PDLLA) is a relatively new subdermal biostimulatory filler that is used for the treatment of deep and shallow facial wrinkles. PDLLA is supplied as lumps of lyophilized powder in vials. It requires reconstitution with sterile water for injection (SWFI) into a homogeneous suspension before administration. The reconstitution methods recommended by the manufacturer are hand-shaking and vortex generator-assisted agitation. However, these methods have some disadvantages. Handshaking agitation, which is used for reconstitution prior to correction of shallow wrinkles (a process that requires $8 \mathrm{~mL}$ of SWFI), is an exhausting process. Vortex generatorassisted agitation, which is used for reconstitution before the correction of deep wrinkles (requiring $1.4 \mathrm{~mL}$ of SWFI), is time-consuming. To address these drawbacks, we propose a simple, quick, effortless, and efficient "back-and-forth" method for the reconstitution of injectable PDLLA. Using this technique, PDLLA can be easily prepared using large or small volumes of SWFI for different purposes related to facial volumization.

Keywords Back-and-forth / Reconstitution / Poly-D,L-lactic acid / AestheFill / Injectable poly-D,L-lactic acid

\section{INTRODUCTION}

Injectable poly-D,L-lactic acid (PDLLA), which was first approved by the Korean Food and Drug Administration in 2014, is a relatively new subdermal biostimulatory filler (AestheFill; REGEN Biotech, Seoul, Korea) [1-3]. It is used for the correction of deep and shallow facial wrinkles, and it is supplied as lumps of lyophilized powder in vials. Reconstituting PDLLA with sterile water for injection (SWFI) to form a homogeneous suspension before injection is crucial to reduce the likelihood of complications.

A vial of injectable PDLLA contains $200 \mathrm{mg}$ of product [2]. Ac-

Received: Nov 19, 2019 Revised: Dec 12, 2019 Accepted: Dec 20, 2019

Correspondence: Chuan-Yuan Lin Tobeauty Aesthetic Clinic, 1F., No. 18-1, Sanmin Rd., Songshan Dist., Taipei 10582, Taiwan

Tel: +886-939642621, Fax: +886-2-27602788,

E-mail: linchuanyuan@doctortou.com

Copyright (๑) 2020 The Korean Society for Aesthetic Plastic Surgery.

This is an Open Access article distributed under the terms of the Creative Commons Attribution Non-Commercial License (https://creativecommons.org/licenses/by-nc/4.0/) which permits unrestricted non-commercial use, distribution, and reproduction in any medium, provided the original work is properly cited. $\quad w w w . e-a a p s . o r g$ cording to the manufacturer's recommendations, $1.4 \mathrm{~mL}$ of SWFI should be used for the treatment of deep wrinkles, whereas $8 \mathrm{~mL}$ of SWFI should be used to treat shallow wrinkles [4]. The recommended reconstitution methods are agitation by hand-shaking or use of a vortex generator. After the desired volume of SWFI is injected into the vial, the mixture must be agitated until the suspension becomes homogeneous. Then, the injectable PDLLA is ready for use. However, if $8 \mathrm{~mL}$ of SWFI is used, it usually takes more than 30 minutes to achieve a homogeneous suspension by handshaking, and the process is exhausting. If $1.4 \mathrm{~mL}$ of SWFI is used, it often takes more than 1 hour to obtain a homogeneous suspension using vortex generator-assisted agitation.

To dissolve all PDLLA lumps more easily and quickly, we propose a novel "back-and-forth" method to reconstitute injectable PDLLA. This is a simple and efficient method to obtain a homogeneous suspension.

\section{IDEA}

We prepared two 10-mL Luer-lock syringes, which we termed the $\mathrm{P}$-syringe and the S-syringe. The plunger of the P-syringe was re- 
moved, as was the rubber stopper of the vial of injectable PDLLA. After the opening of the vial was wiped with alcohol-absorbed cotton, the P-syringe and vial were held mouth-to-mouth. Because the sizes of the openings of the vial and the P-syringe were similar, no gap existed between them. The PDLLA lumps were transferred smoothly by pouring them from the vial into the P-syringe. The plunger was reinserted into the P-syringe, after which $8 \mathrm{~mL}$ of SWFI was retrieved into the $\mathrm{S}$-syringe. The two syringes were tightly connected to a three-way stopcock by their Luer-lock tips (Fig. 1). The plunger of the S-syringe was then slowly pushed so that SWFI

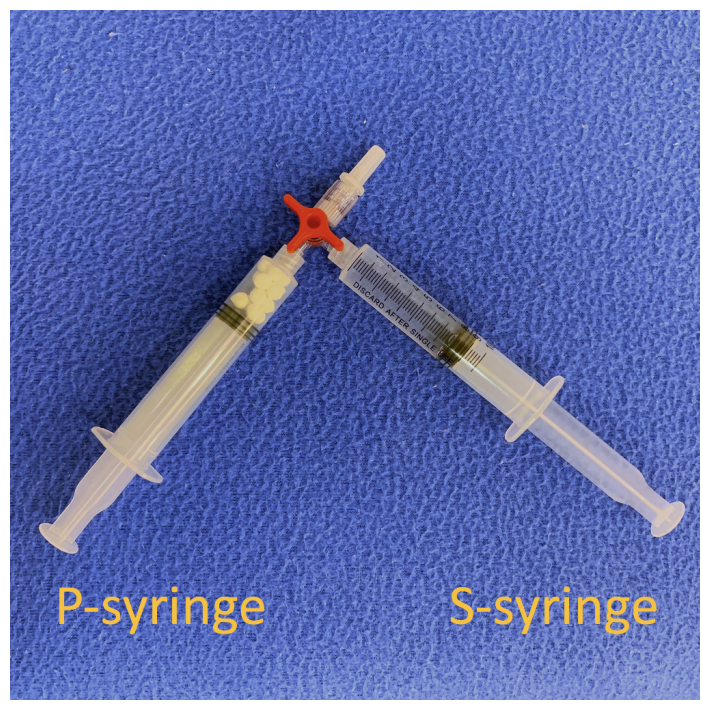

Fig. 1. P-syringe containing poly-D,L-lactic acid lumps and S-syringe containing $8 \mathrm{~mL}$ sterile water for injection. These syringes were tightly connected to a three-way stopcock by their Luer-lock tips. could be injected into the P-syringe. The P-syringe was placed in an upright position with the stopcock at the top, and the plunger of the P-syringe was slowly pushed until air was expelled into the Ssyringe. Then, the S-syringe was disconnected from the stopcock so that air could be expelled from the syringe, after which the S-syringe was reconnected to the stopcock. The PDLLA lumps were immersed in the P-syringe for 3-5 minutes to allow them to absorb water and swell (Fig. 2). Then, the P-syringe was placed in an upright position with the stopcock at the bottom (Fig. 3A). The swollen PDLLA lumps floated within the upper part of the P-syringe.

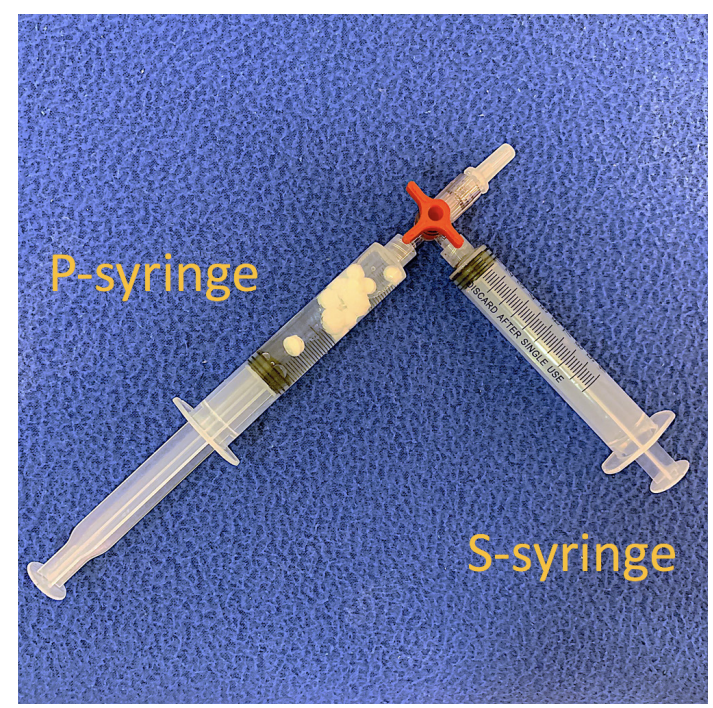

Fig. 2. Injection of the sterile water for injection into the P-syringe; the poly-D,L-lactic acid lumps were allowed to soak for 3 minutes.
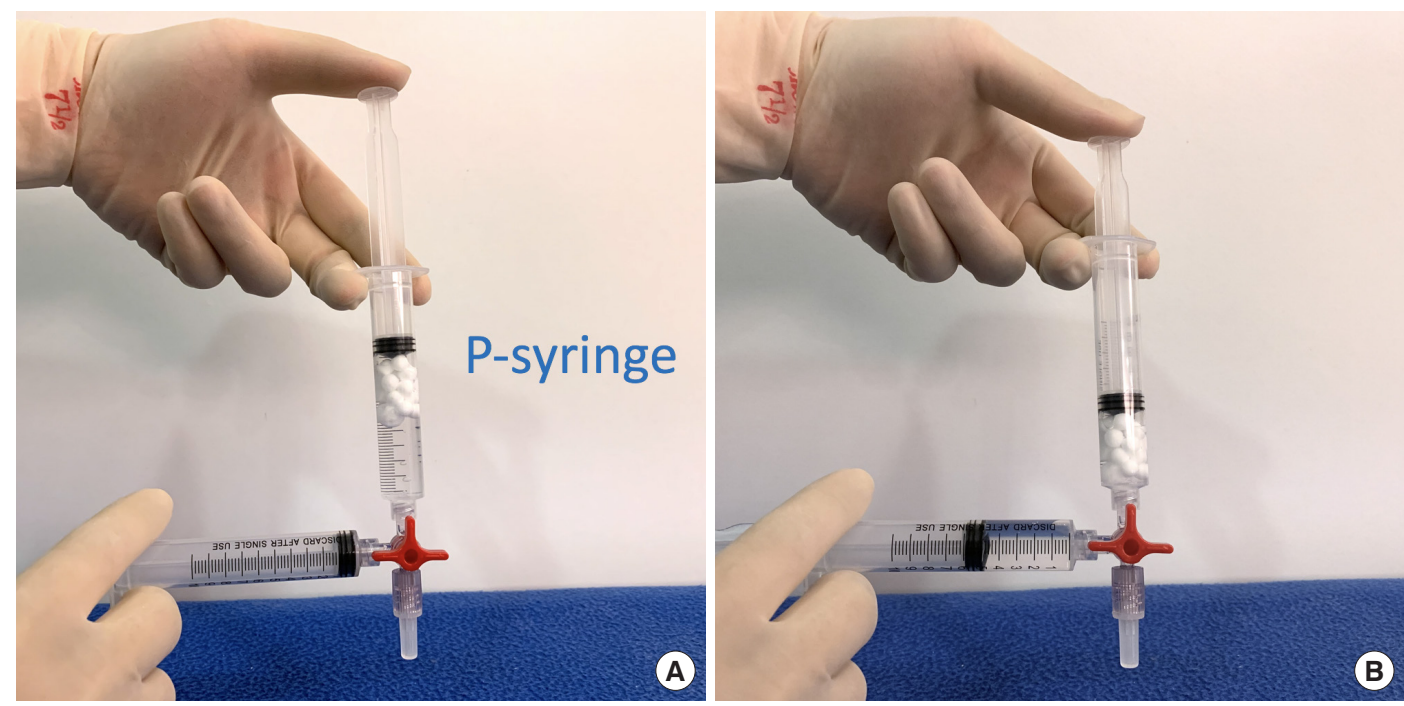

Fig. 3. (A) Placement of the P-syringe in an upright position with the stopcock at the bottom. (B) Pushing of the plungers of these two syringes back-and-forth gradually. 

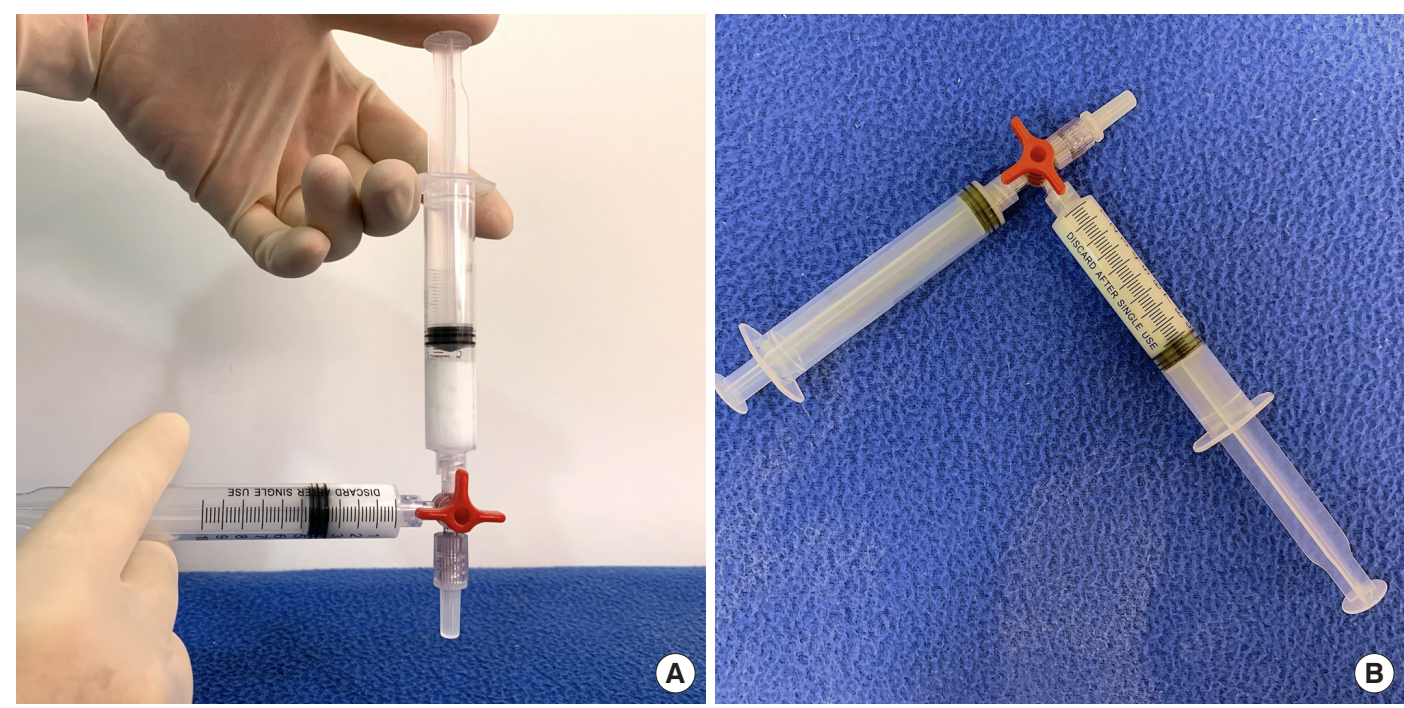

Fig. 4. (A) Back-and-forth continuous pushing between the two syringes, until the (B) total dissolution of all poly-D,L-lactic acid lumps into a homogeneous suspension.

SWFI was gradually pushed back-and-forth between the two syringes (Fig. 3B). Occasionally, a clog occurred at the connection between the stopcock and the P-syringe. To solve this problem, we disconnected the S-syringe from the stopcock, drew air, reconnected the S-syringe to the stopcock, and then pushed the air into the P-syringe. Afterwards, the back-and-forth pushing between the two syringes was repeated (Fig. 4A) until all of the PDLLA lumps were completely dissolved into a homogeneous suspension (Fig. 4B, Supplemental Digital Content 1). The amount of time required for the total dissolution of all PDLLA lumps into a homogeneous suspension when utilizing back-and-forth pushing usually fell within 2 minutes. Including the 3 - to 5 -minute immersion time, the total reconstitution time required when using this method is approximately 5-7 minutes. Before injection, we used another syringe to retrieve $2 \mathrm{~mL}$ of $1 \%$ or $2 \%$ lidocaine by connecting this syringe to the three-way stopcock and injecting its contents into the suspension. Then, the back-and-forth method was repeated for $10-20$ seconds to mix the suspension and the lidocaine. The suspension was then ready to use.

This back-and-forth reconstitution method for the preparation of injectable PDLLA can be used with as little as $1.4 \mathrm{~mL}$ of SWFI, and reconstitution of multiple vials of injectable PDLLA using the back-and-forth method is also feasible. The differences between these uses and the previously-described method are that when a small volume of SWFI is used, 5-8 minutes of immersion time may be needed for absorption of water by the PDLLA lumps, and when multiple vials of injectable PDLLA are reconstituted simultaneously, larger Luer-lock syringes should be used. Irrespective of the volume of SWFI and the number of vials of injectable PDLLA used, the total reconstitution time should be approximately 5-10 minutes using this back-and-forth method.

\section{DISCUSSION}

Compared with administration of injectable poly-L-lactic acid (PLLA; Sculptra; Galderma, Fort Worth, TX, USA), injectable PDLLA administration is associated with less frequent appearance of subcutaneous papules and nodules [2,5-11]. Injectable PLLA consists of 40- to 63- $\mu \mathrm{m}$ irregular solid PLLA microparticles, carboxymethyl cellulose, and non-pyrogenic mannitol. Injectable PLLA is available in vials in the form of lyophilized powder cakes, and it also requires reconstitution with SWFI to form a homogeneous suspension prior to injection. Adverse reactions associated with injectable PLLA include the formation of subcutaneous papules and nodules, and one reason for such complications is the insufficient reconstitution of injectable PLLA. To solve this problem, the consensus on the reconstitution of injectable PLLA is that the operator should use a suitable quantity of SWFI $(7-8 \mathrm{~mL})$, allow adequate hydration time ( $\geq 24$ hours), and not shake the vial during hydration [12]. If these recommendations are not followed, PLLA clumps may persist, which can lead to papule and nodule formation.

Injectable PDLLA consists of 30- to 70- $\mu \mathrm{m}$ multi-porous PDLLA microspheres and carboxymethyl cellulose [2,3]. In 2012, clinical trials with injectable PDLLA were performed in Korea. In that randomized, evaluator-blinded, and comparative study, the injectable PDLLA was reconstituted using $0.7 \mathrm{~mL}$ of SWFI for every $100 \mathrm{mg}$ of injectable PDLLA, which corresponds to $1.4 \mathrm{~mL}$ of SWFI for each vial of injectable PDLLA $[2,5,6]$. The product was injected into the nasolabial folds to assess its efficacy and safety. The study showed that injectable PDLLA is not inferior to hyaluronic acid in the correction of moderate to severe nasolabial folds. Among the 30 patients who received PDLLA injections, one experienced skin discoloration caused by bruising, one displayed small transient vesicle 
formation, and two reported a slight mass sensation at the injection site. However, these complications spontaneously resolved without any treatment by the 2-month follow-up. The self-limiting nature and the short duration of the nodules suggest that they are generally caused by the injection procedure rather than being product-related complications or hypersensitivity responses [5]. This result is consistent with our clinical experience, wherein nodule formation seldom occurred after administration of injectable PDLLA. However, we believe that the reconstitution of injectable PDLLA into a homogeneous suspension is crucial to prevent this problem.

The back-and-forth method for the reconstitution of injectable PDLLA has many benefits over the previous hand-shaking and vortex generator-assisted agitation methods. The back-and-forth method is simple and fast, does not require a vortex generator, results in a more homogeneous suspension, allows for the reconstitution of multiple vials in one large syringe, is an easier way to divide product into multiple smaller injection syringes, and results in less waste of product. These benefits are particularly obvious when a smaller volume of SWFI is used for the reconstitution of injectable PDLLA. When reconstituting injectable PDLLA with a small volume of SWFI, it was difficult to obtain a homogeneous suspension using the previous hand-shaking agitation method, therefore necessitating the use of the time-consuming vortex generator-assisted method. Otherwise, the suspension of injectable PDLLA reconstituted with this small volume of SWFI would be very sticky. The suspension would then adhere to the wall or even to the rubber stopper of the vial, and it would be very difficult to draw all of the suspension from the vial. When using the back-and-forth reconstitution method, all of the PDLLA is already in the syringe for use.

However, the back-and-forth method has some disadvantages. When transferring PDLLA lumps from the vial to the syringe, the chances of contamination increase, and accidental spilling of the PDLLA lumps may occur. Therefore, this procedure should be performed by a well-trained professional under strict aseptic conditions. By following these rules, in our 2 years of clinical experience using this back-and-forth method, we have encountered no complications such as infection, nodule formation, or granuloma formation. The connection between the two syringes and the stopcock should be tightly bound using Luer-lock tips. This prevents the accidental dislodgement of one syringe from the stopcock due to the high pressure caused by the back-and-forth procedure, thus avoiding spilling of the contents and preventing waste.

We chose a three-way stopcock to connect the two syringes because the two syringes can then be connected at a $90^{\circ}$ angle, which allows for easier operation with two hands than a $180^{\circ}$ angle. Additionally, the through hole of the three-way stopcock is smaller than that of the two-way stopcock, meaning that the injection force between the two syringes is stronger with the three-way than the twoway stopcock; the third connecting port can also be used for other purposes, such as lidocaine addition or product division.
In conclusion, this novel back-and-forth reconstitution method is simple, quick, effortless, and efficient. Using this technique, injectable PDLLA can be prepared with either large or small volumes of SWFI for various purposes related to facial volumization.

\section{NOTES}

\section{Conflict of interest}

JYL and CYL are medical directors of REGEN Biotech. No other potential conflict of interest relevant to this article was reported.

\section{ORCID}

Se-Yi Chen

https://orcid.org/0000-0002-6872-5601

Jui-Yu Lin

https://orcid.org/0000-0003-3040-8982

Chuan-Yuan Lin

https://orcid.org/0000-0003-2306-478X

\section{Supplemental material}

Supplementary materials can be found via https://doi.org/10.14730/ aaps.2019.01977

\section{REFERENCES}

1. Lin CY, Lin JY, Yang DY, et al. Efficacy and safety of poly-D,L-lactic acid microspheres as subdermal fillers in animals. Plast Aesthet Res 2019;6:16.

2. Chen SY, Lin JY, Lin CY. Compositions of injectable poly-D,L-lactic acid and injectable poly-L-lactic acid. Clin Exp Dermatol 2020;45:347-8.

3. Chen SY, Lin JY, Lin CY. Letter to the editor regarding the article "Kwon T-R, Han SW, Yeo IK, et al. Biostimulatory effects of polydioxanone, poly-D,L lactic acid, and polycaprolactone fillers in mouse model. J Cosmet Dermatol 2019;18(4):1002-1008." J Cosmet Dermatol 2020; 19:1004-5.

4. AestheFill (new generation facial wrinkle filler): package insert. Seoul: REGEN Biotech, Inc.; 2014.

5. Hyun MY, Lee Y, No YA, et al. Efficacy and safety of injection with poly-L-lactic acid compared with hyaluronic acid for correction of nasolabial fold: a randomized, evaluator-blinded, comparative study. Clin Exp Dermatol 2015;40:129-35.

6. No YA, Seok J, Hyun MY, et al. Long-term (24-month) safety evaluation of poly-DL-lactic acid filler injection for the nasolabial fold: a multicenter, open, randomized, evaluator-blind, active-controlled design. Plast Reconstr Surg 2015;135:1074e-1075e.

7. Lorenc ZP, Greene T, Gottschalk RW. Injectable poly-L-lactic acid: understanding its use in the current era. J Drugs Dermatol 2016;15:75962.

8. Vleggaar D, Fitzgerald R, Lorenc ZP. Understanding, avoiding, and treating potential adverse events following the use of injectable polyL-lactic acid for facial and nonfacial volumization. J Drugs Dermatol 2014;13(4 Suppl):s35-9.

9. Vleggaar D, Fitzgerald R, Lorenc ZP. The history behind the use of in- 
jectable poly-L-lactic acid for facial and nonfacial volumization: the positive impact of evolving methodology. J Drugs Dermatol 2014;13(4 Suppl):s32-4.

10. Alessio R, Rzany B, Eve L, et al. European expert recommendations on the use of injectable poly-L-lactic acid for facial rejuvenation. J Drugs Dermatol 2014;13:1057-66.
11. Palm MD, Woodhall KE, Butterwick KJ, et al. Cosmetic use of poly-Llactic acid: a retrospective study of 130 patients. Dermatol Surg 2010; 36:161-70.

12. Vleggaar D, Fitzgerald R, Lorenc ZP, et al. Consensus recommendations on the use of injectable poly-L-lactic acid for facial and nonfacial volumization. J Drugs Dermatol 2014;13(4 Suppl):s44-51. 